\title{
Class-Uniformly Resolvable Group Divisible Structures I: Resolvable Group Divisible Designs
}

\author{
Peter Danziger* \\ Department of Mathematics, Physics and Computer Science \\ Ryerson Polytechnic University \\ Toronto, ON M5B 2K3, Canada \\ danziger@acs.ryerson.ca \\ Brett Stevens $^{\dagger}$ \\ School of Mathematics and Statistics \\ Carleton University \\ 1125 Colonel By Dr. \\ Ottawa ON K1S 5B6, Canada \\ brett@math.carleton.ca
}

Submitted: Jun 3, 2003; Accepted: Mar 15, 2004; Published: Mar 25, 2004

MR Subject Classifications: 05B05, 05B40

\begin{abstract}
We consider Class-Uniformly Resolvable Group Divisible Designs (CURGDD), which are resolvable group divisible designs in which each of the resolution classes has the same number of blocks of each size. We derive the fully general necessary conditions including a number of extremal bounds. We present some general constructions including a novel construction for shrinking the index of a master design. We construct a number of infinite families, primarily with block sizes 2 and $k$, including some extremal cases.
\end{abstract}

\section{Introduction}

Class-Uniformly Resolvable incidence structures, where each resolution class has the same number of blocks of each size are discussed in [3, 4, 5, 12]. These references contain motivations, applications and discussions of related objects. We will assume that the reader is acquainted with design theory terminologies and we refer them to [2]. In this article we investigate class-uniformly resolvable group divisible designs

${ }^{*}$ Supported by NSERC discovery grant \#OGP0170220.

${ }^{\dagger}$ Supported by PIMS, MITACS and IBM Watson Research and NSERC.

THE ELECTRONIC JOURNAL OF COMBINATORICS 11 (2004), \#R23 
Definition 1.1. A Class-Uniformly Resolvable Group Divisible Design, CURGDD $D_{\lambda}$, of type $\prod g^{u_{g}}$ with partition $\prod k^{p_{k}}$ is a $\mathrm{GDD}_{\lambda}$ with the additional property that the blocks can be partitioned into resolution classes with partition $\prod k^{p_{k}}$.

CURGDDs were introduced by Lamken et al. and were used by Wevrick and Vanstone to construct CURDs [5, 12]. A CURGDD with all $g=1$ is a CURD. Also a CURD with partition $k_{1}^{p_{k_{1}}} \ldots k_{n}^{p_{k_{n}}}$ and one resolution class identified as the groups is a CURGDD of type $k_{1}^{p_{k_{1}}} \ldots k_{n}^{p_{k_{n}}}$ and with partition $k_{1}^{p_{k_{1}}} \ldots k_{n}^{p_{k_{n}}}$. The dual of a CURGDD is a resolvable packing array or transversal packing $[10,11]$.

Example 1.2. We present a CURGDD of type $6^{3}$ with partition $2^{6} 3^{2}$ with 9 resolution classes. The point set is $\mathbb{Z}_{18}$. The groups are

$$
\{0,1,2,3,4,5\},\{6,7,8,9,10,11\},\{12,13,14,15,16,17\} .
$$

The following nine rows are the resolution classes.

$\begin{array}{llllllll}\{17,0,7\} & \{16,1,8\} & \{4,9\} & \{5,12\} & \{10,15\} & \{2,6\} & \{13,3\} & \{11,14\} \\ \{12,1,6\} & \{2,7,14\} & \{13,4\} & \{16,3\} & \{15,8\} & \{9,17\} & \{11,0\} & \{5,10\} \\ \{9,13,2\} & \{5,11,16\} & \{7,3\} & \{15,1\} & \{4,12\} & \{14,8\} & \{6,0\} & \{10,17\} \\ \{11,13,1\} & \{8,17,3\} & \{9,16\} & \{12,2\} & \{15,6\} & \{4,7\} & \{14,5\} & \{10,0\} \\ \{9,3,14\} & \{7,15,5\} & \{13,0\} & \{12,11\} & \{1,10\} & \{17,2\} & \{6,16\} & \{4,8\} \\ \{6,13,5\} & \{14,4,10\} & \{1,9\} & \{2,8\} & \{17,11\} & \{0,16\} & \{12,7\} & \{15,3\} \\ \{12,3,10\} & \{9,15,0\} & \{2,11\} & \{6,14\} & \{5,17\} & \{16,4\} & \{1,7\} & \{8,13\} \\ \{16,2,10\} & \{4,11,15\} & \{8,5\} & \{14,0\} & \{12,9\} & \{17,1\} & \{3,6\} & \{13,7\} \\ \{8,12,0\} & \{4,6,17\} & \{10,13\} & \{1,14\} & \{2,15\} & \{11,3\} & \{9,5\} & \{7,16\}\end{array}$

In Section 2 we derive the necessary conditions for the existence of CURGDDs and discuss resulting bounds on their size. Next, in Section 3, we present constructions which will be used to produce infinite families and give illustrative examples of these results in practice. In particular we present a novel construction that reduces the index by increasing the group size. We use this construction to produce a large family of CURGDDs with block sizes 2 and $k$, including some extremal cases. We will make frequent use of several families of designs: 3-RGDDs, 4 -RGDDs, $\operatorname{RTD}(2, g), \operatorname{RTD}(3, g)$ and $\operatorname{RTD}(4, g)[1,2,6,7,8,9]$. In Section 4 we construct a number of infinite families of CURGDDs with block sizes 2 and 3 and block sizes 3 and 4 . Finally we provide some concluding remarks.

\section{Necessary Conditions}

\subsection{The General Case}

In this section we discuss the general necessary conditions for the existence of a CURGDD of type $\prod g^{u_{g}}$ with partition $\prod k^{p_{k}}$. They are derived in a similar manner to those in [4] and we refer the reader there for details. Letting $r_{k}(x)$ be the number of blocks of size $k$ 
which contain $x$ we have

$$
\begin{aligned}
\sum_{k} k p_{k} & =\sum_{j=1}^{m} g u_{g}=v \\
r \sum_{k} k(k-1) p_{k} & =\lambda\left(v^{2}-\sum_{1 \leq i \leq m} g^{2} u_{g}\right), \\
\sum_{k} r_{k}(x)(k-1) & =\lambda(v-g)
\end{aligned}
$$

where $x$ appears on a group of size $g$. In the case that all groups are the same size these simplify considerably.

\subsection{Two Block Sizes}

We now consider the case where there are only two block sizes, $k$ and $l$ which we treat as interchangeable, except where noted. We obtain a number of extra conditions, including some extremal bounds.

$$
\begin{aligned}
l p_{l} & \equiv v \bmod k \\
r_{k}(x) & =\frac{r(l-1)-\lambda(v-g)}{l-k} \\
v & \leq \lambda\left|\frac{\alpha_{l}^{2}}{d(k-1)}-\frac{(k-1) \sum_{1 \leq i \leq m} g^{2} u_{g}}{d}\right|-\frac{\alpha_{l}}{k-1}
\end{aligned}
$$

Where $\alpha_{l}=l(l-k) p_{l}$ and $d=\operatorname{gcd}\left(r(k-1)^{2}, \lambda v(k-1)-\lambda \alpha_{l}\right)$. When all group sizes are the same, Equation 5 implies that each point appears in the same number of blocks of each size. To illustrate that this property does not generalize to more than two block sizes we refer the reader to the examples given in [4]. These may be used as ingredients in Theorem 3.1 to give examples with constant $g>1$.

In the case of a CURGDD of type $g^{u}$ with partition $k^{p_{k}} l^{p_{l}}$ we get

$$
g u \leq \lambda\left|\frac{\alpha_{l}}{d^{\prime}}\left(\frac{\alpha_{l}}{k-1}+g\right)\right|-\frac{\alpha_{l}}{k-1}
$$

where $d^{\prime}=\operatorname{gcd}\left(r(k-1)^{2}, \lambda\left(g(u-1)(k-1)-\alpha_{l}\right)\right)$. When $\alpha_{l}=-(k-1) g$ then no bound is derived from Equation 7 and $g u=(k-1) r / \lambda$. 
When the blocks are size 2 and 3 these reduce to

$$
\begin{aligned}
2 p_{2}+3 p_{3} & =\sum_{j=1}^{m} g u_{g}=v, \\
2 r\left(p_{2}+3 p_{3}\right) & =\lambda\left(v^{2}-\sum_{1 \leq i \leq m} g^{2} u_{g}\right), \\
r_{2}(x) & =2 r-\lambda(v-g), \\
r_{3}(x) & =\lambda(v-g)-r, \\
p_{3} & \equiv v \bmod 2, \\
p_{2} & \equiv-v \bmod 3, \\
v & \leq \frac{\lambda}{d_{1}}\left|9 p_{3}^{2}-\sum_{1 \leq i \leq m} g^{2} u_{g}\right|-3 p_{3}, \\
v & \leq \frac{\lambda}{d_{2}}\left|p_{2}^{2}-\sum_{1 \leq i \leq m} g^{2} u_{g}\right|+p_{2},
\end{aligned}
$$

where $x$ appears on a group of size $g, d_{1}=\operatorname{gcd}\left(r, \lambda\left(v-3 p_{3}\right)\right)$ and $d_{2}=\operatorname{gcd}\left(2 r, \lambda\left(v+p_{2}\right)\right)$.

When all group sizes are equal we can further simplify Inequalities 12 and 13 to

$$
\begin{aligned}
g u & \leq \frac{3 \lambda p_{3}\left(3 p_{3}+g\right)}{d_{1}^{\prime}}-3 p_{3}, \\
g u & \leq \frac{\lambda p_{2}\left|p_{2}-g\right|}{d_{2}^{\prime}}+p_{2},
\end{aligned}
$$

where $d_{1}^{\prime}=\operatorname{gcd}\left(r, \lambda\left(g(u-1)-3 p_{3}\right)\right)$ and $d_{2}^{\prime}=\operatorname{gcd}\left(2 r, \lambda\left(g(u-1)+p_{2}\right)\right)$. When $p_{2}=g$ then no bound is derived from Equation 15 and $g u=2 r / \lambda$.

\section{General Constructions}

We now consider some general constructions for CURGDDs. The first two are analogues of Wilson's constructions [13] adapted to CURGDDs.

Theorem 3.1 (Wilson's Fundamental Construction). Suppose there exists a $K$ $R G D D_{\lambda_{1}}$ (the master) of type $\prod_{i=1}^{m} g$ with groups $G_{i}, 1 \leq i \leq m$ with resolution classes $R_{j}, 1 \leq j \leq r$. To each point $x$ assign a weight $w(x)$, and suppose that for each $b_{l} \in R_{j}$ there is a CURGDD $D_{\lambda_{2}}$ (an ingredient) of type $\prod_{x \in B_{l}} w(x)$ with partition $\prod k^{q_{l k}}$ and $r_{j}$ resolution classes, where

$$
\sum_{b_{l} \in R_{j}} q_{l k}=p_{k}
$$

for every $j$. Then there exists a CURGDD $D_{\lambda_{1} \lambda_{2}}$ of type $\prod_{i=1}^{m}\left(\sum_{x \in G_{i}} w(x)\right)$, with partition $\prod k^{p_{k}}$ with $\sum_{j=1}^{r} r_{j}$ resolution classes. 
Proof. This is Wilson's construction [13]. We note that for each resolution class, $R_{j}$, in the master, the resolution classes from the ingredients will form resolutions of the whole set, with partition

$$
\prod k^{\sum_{b_{l} \in R_{j}} q_{l k}}=\prod k^{p_{k}}
$$

CURGDDs with fixed $r$ and RTDs are ideal ingredients for this construction.

Theorem 3.2 (Breaking up the Groups). If there exists a CURGDD $D_{\lambda}$ of type $(n g)^{u}$ with partition $\prod k^{p_{k}}$ with $r$ resolution classes and a CURGDD $D_{\lambda}$ of type $g^{n}$ and partition $\prod k^{q_{k}}, r^{\prime}$ resolution classes, and $u q_{k}=p_{k}$ for all $k$, then there exists a CURGDD of type $g^{n u}$ with partition $\prod k^{p_{k}}$ and $r+r^{\prime}$ resolution classes.

Proof. Place a copy of the smaller CURGDD on each group of the larger.

We now present our main construction which strikingly, allows us to reduce the index of a CURGDD ${ }_{\lambda}$, whilst increasing the group size. We start by introducing some notation.

Given a set of blocks $\mathcal{B}$ over a point set $X$, we call a block maximal with respect to a pair $i, j \in X$, where $i \neq j$, if it is a largest block containing the pair $i, j$. Let $\mathcal{M} \subseteq \mathcal{B}$ be the set of maximal blocks, i.e. $\mathcal{M}=\{B \in \mathcal{B} \mid B$ is maximal for some pair $i, j \in X\}$. If for every $M \in \mathcal{M}$ and every $B \in \mathcal{B}$, either $B \subseteq M$ or $|M \cap B| \leq 1$, then we say that $\mathcal{B}$ is maximally contained. If $\mathcal{B}$ is maximally contained then a maximal block is maximal for every pair contained within it and every pair is contained in a unique member of $\mathcal{M}$.

If $\mathcal{B}$ is a maximally contained design with index $\lambda$ then $\mathcal{M}$ is a design on $X$ with index 1. Further, for each $M \in \mathcal{M}$ the collection of blocks $\mathcal{D}_{M}=\{B \in \mathcal{B} \mid B \subseteq M\}$ forms a design of index $\lambda$ with point set $M$. Given $\lambda^{\prime}$ with $\lambda^{\prime} \mid \lambda$, if for each $M \in \mathcal{M}, \mathcal{D}_{M}$ can be decomposed into $\lambda / \lambda^{\prime}$ designs each with index $\lambda^{\prime}$, then we call $\mathcal{B} \lambda^{\prime}$-maximally contained. In particular, if $\lambda^{\prime}=1$ then for every $M \in \mathcal{M}, \mathcal{D}_{M}$ can be decomposed into $\lambda$ designs of index 1. These designs are either the trivial design consisting of a single block containing all the points of $M$, or they consist of non maximal blocks. Every block of $\mathcal{B}$ appears in exactly one such sub-design with index 1 .

Theorem 3.3 ( $\lambda$ Blow-up). If there exists a 1-maximally contained CURGDD $D_{\lambda}$ of type $g^{u}$ with partition $\prod k^{p_{k}}$ and for each maximal block $M$ there exists an $\operatorname{RTD}_{\lambda_{1}}(|M|, \lambda)$ then there exists a CURGDD $D_{\lambda_{1}}$ of type $(\lambda g)^{u}$ and partition $\prod k^{\lambda p_{k}}$.

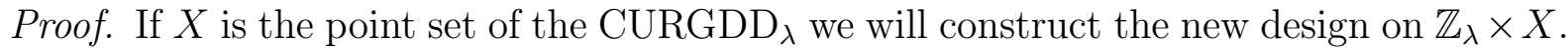
Blow up the points of the $\mathrm{CURGDD}_{\lambda}$ by $\lambda$ and place upon each blown up maximal block, $M$, an $\operatorname{RTD}_{\lambda_{1}}(|M|, \lambda)$. Since $\mathcal{M}$ is itself a GDD the result is a GDD by Wilson's construction.

Partition the $\lambda_{1} \lambda$ resolution classes of the $\operatorname{RTD}_{\lambda_{1}}(|M|, \lambda)$ on $M$ into $\lambda$ sets of $\lambda_{1}$ resolution classes of $\mathbb{Z}_{\lambda} \times M, \mathcal{R}_{0}, \ldots, \mathcal{R}_{\lambda-1}$. Since the CURGDD is 1-maximally contained, $\mathcal{D}_{M}$ can be decomposed into $\lambda$ sub-designs with index $1, \mathcal{D}_{0}, \ldots, \mathcal{D}_{\lambda-1}$.

For each resolution class $R_{j} \in \mathcal{R}_{i}$ of $\mathbb{Z}_{\lambda} \times M, 0 \leq i<\lambda, 0 \leq j<\lambda_{1}$, we cover the blocks of $R_{j}$ with copies of $\mathcal{D}_{i}$. For each block $B_{i} \in \mathcal{D}_{i}$ we use $B_{i j}$ to denote the blocks 
formed by applying $B_{i}$ to $R_{j}$, we note that each $B_{i j}$ consists of $\lambda$ blocks of size $|B|$, which form a resolution of $\mathbb{Z}_{\lambda} \times B$.

If $S_{l}, 0 \leq l<r$ are the resolution classes of the original CURGDD $\mathrm{CU}_{\lambda}$ then

$$
T_{j l}=\bigcup_{B_{i} \in S_{l}} B_{i j}
$$

where $0 \leq j<\lambda_{1}$, form resolutions of the expanded design with partition $\prod k^{\lambda p_{k}}$.

Essentially Theorem 3.3 allows us to perform the same construction as Theorem 3.1 even in situations where we do not have the desired master CURGDD. When there is only

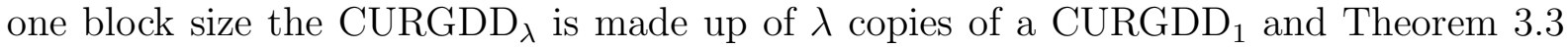
becomes an instance of Theorem 3.1 using RTDs as ingredients. This is because the set of maximal blocks forms a design of index 1 and all other blocks must be part of a sub-design contained within one of these. Multiple block sizes is the essential ingredient that makes Theorem 3.3 a potent generalization of Theorem 3.1. We illustrate Theorem 3.3 with the following example.

Example 3.4. We construct a CURGDD $\mathrm{CH}_{5}$ of type $1^{7}$ with partition $2^{2} 3^{1}$ on $\mathbb{Z}_{7}$ with 21 resolution classes, by developing the following three resolution classes additively:

$$
\begin{array}{lll}
\{0,1,3\} & \{2,4\} & \{5,6\} \\
\{0,1,3\} & \{2,5\} & \{4,6\} \\
\{0,1,3\} & \{2,6\} & \{4,5\} .
\end{array}
$$

This CURGDD satisfies the hypotheses of Theorem 3.3, since all triples either intersect in three points or only one point. Therefore Theorem 3.3 gives the existence of a CURGDD 1 of type $5^{7}$ with partition $2^{10} 3^{5}$. The set of points is $\mathbb{Z}_{5} \times \mathbb{Z}_{7}$, the groups are $\{(0, i),(1, i),(2, i),(3, i),(4, i)\}$, for $0 \leq i \leq 6$.

We now show the construction in action for a given maximal block, say $\{0,1,3\}$. We consider all the blocks that its point set induces:

$$
\{0,1,3\},\{0,1,3\},\{0,1,3\},\{0,1\},\{0,1\},\{0,3\},\{0,3\},\{1,3\},\{1,3\} \text {. }
$$

$\mathcal{D}_{0}=\mathcal{D}_{1}=\mathcal{D}_{2}=\{0,1,3\}, \mathcal{D}_{3}=\mathcal{D}_{4}=\{\{0,1\},\{0,3\},\{1,3\}\}$. We take an $\operatorname{RTD}(3,5)$ and place three of its resolution classes on the three blown up blocks of the form $\{0,1,3\}$, indexing its three groups by the three points 0,1 , and 3 . We will assume that the remaining two resolution classes of the $\operatorname{RTD}(3,5)$ are

$$
\begin{aligned}
& \{(0,0),(0,1),(0,3)\} \quad\{(0,0),(1,1),(2,3)\} \\
& \{(1,0),(1,1),(1,3)\} \quad\{(1,0),(2,1),(3,3)\} \\
& \mathcal{R}_{3}=\{(2,0),(2,1),(2,3)\}, \mathcal{R}_{4}=\{(2,0),(3,1),(4,3)\} \\
& \{(3,0),(3,1),(3,3)\} \quad\{(3,0),(4,1),(0,3)\} \\
& \{(4,0),(4,1),(4,3)\} \quad\{(4,0),(0,1),(1,3)\} .
\end{aligned}
$$

We now use $\mathcal{D}_{3}$ and $\mathcal{D}_{4}$ respectively to decompose these classes. Thus, for example, the block $\{(0,0),(0,1),(0,3)\}$ of $\mathcal{R}_{3}$ generates the blocks $\{(0,0),(0,1)\},\{(0,0),(0,3)\}$, 
$\{(0,1),(0,3)\}$. Considering the blocks of the form $\{0,1\}, B_{3}$ and $B_{4}$ say, from $\mathcal{D}_{3}$ and $\mathcal{D}_{4}$, we will create two induced resolution classes of $\mathbb{Z}_{5} \times\{0,1\}$ from those above:

$$
\begin{aligned}
& \{(0,0),(0,1)\} \quad\{(0,0),(1,1)\} \\
& \{(1,0),(1,1)\} \quad\{(1,0),(2,1)\} \\
& B_{30}=\{(2,0),(2,1)\}, \quad B_{40}=\{(2,0),(3,1)\} \\
& \{(3,0),(3,1)\} \quad\{(3,0),(4,1)\} \\
& \{(4,0),(4,1)\} \quad\{(4,0),(0,1)\} \text {. }
\end{aligned}
$$

We proceed similarly with the remaining blocks contained in $\{0,1,3\}$ and repeat the process for the other maximal blocks.

The resolution classes in the final CURGDD are obtained from the blow up of the resolution classes of the original CURGDD. Thus, for example, the two classes $B_{30}$ and $B_{40}$ above will form part of the resolution class obtained from the blow up of the resolution classes

$$
\begin{aligned}
& \{2,3,5\},\{4,6\},\{0,1\} \\
& \{3,4,6\},\{5,2\},\{0,1\}
\end{aligned}
$$

of the original CURGDD respectively.

Theorem 3.5. For $q$ a prime power, $2 \leq k \leq q, 0 \leq m \leq q /(k-1)$, there exists a CURGDD $D_{q-m(k-2)}$ of type $q^{k}$ with partition $2^{m k(k-1) / 2} k^{q-m(k-1)}$, satisfying the conditions of Theorem 3.3.

Proof. Let $U=\left\{g_{0}, g_{1} \ldots g_{k-1}\right\} \subseteq G F(q)$ be a set of cardinality $k$, where $g_{0}=0$, and let $V_{0} \subseteq G F(q)$ be a set of cardinality $q-m(k-1)$. We will construct the design with point set $G F(q) \times U$, where the groups are given by $G_{i}=G F(q) \times i$ for each $i \in U$. For each $x \in G F(q)$ we create $q-m(k-1)$ disjoint blocks of size $k$ :

$$
B_{x}=\left\{\left\{\left(v+g_{i} x, g_{i}\right) \mid g_{i} \in U\right\} \mid v \in V_{0}\right\} .
$$

Partition $G F(q) \backslash V_{0}$ into $m$ sets, $V_{j}$ for $1 \leq j \leq m$, of cardinality $k-1$. We index the elements of each $V_{j}$ as $x_{j n}$ for $1 \leq n \leq k-1$. For each $j$ and each $x \in G F(q)$ we create the following set of disjoint pairs:

$$
P_{j x}=\left\{\left\{\left(x_{j n}+g_{i} x, g_{i}\right),\left(x_{j(i+1)}+g_{n} x, g_{n}\right)\right\} \mid 0 \leq i \leq k-1, i+1 \leq n \leq k-1\right\},
$$

where $g_{i}, g_{n} \in U$.

The collections of sets, $R_{x}=B_{x} \cup\left(\bigcup_{j} P_{j x}\right)$ form $q$ resolution classes of $G F(q) \times U$. The collection $S=\bigcup_{x} R_{x}$ can be developed additively over $G F(q)$ in the first coordinate to obtain the design. The general form of $R_{0}$ is shown in Figure 1. 
Figure 1: $R_{0}$ for $q=11, m=1$ and $k=7$.

$k$

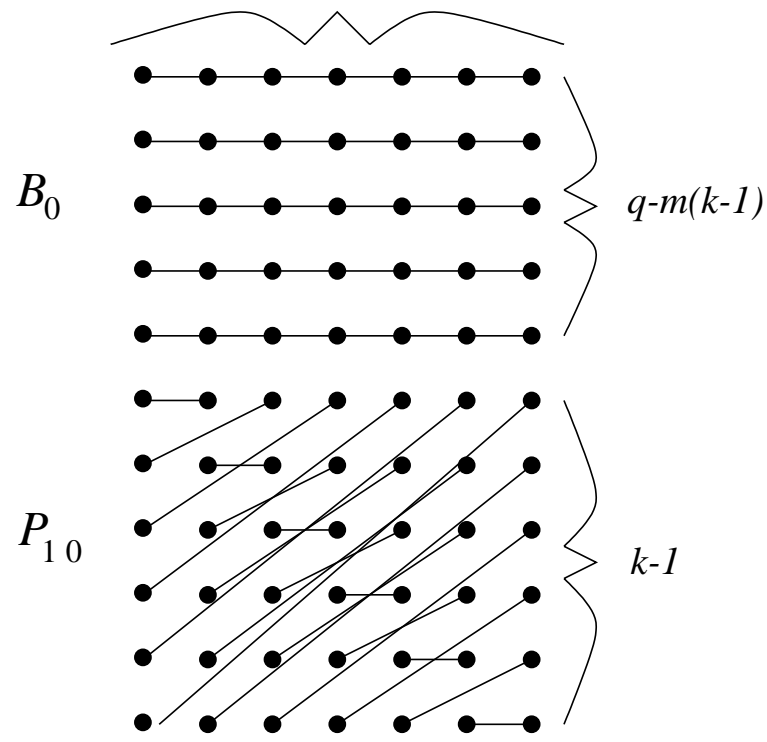

Example 3.6. There exists a CURGDD 4 of type $5^{3}$ with partition $2^{3} 3^{3}$ satisfying the hypotheses of Theorem 3.3.

We take $k=3$ and $m=1$ in Theorem 3.5. $U=V_{0}=\{0,1,2\}$, the set of points is $G F(5) \times\{0,1,2\}$, the groups are $\{(0, i),(1, i),(2, i)(3, i)(4, i)\}$ for $0 \leq i \leq 2$. The resolution classes are, for each $i$ and each $x \in G F(5)$ :

$$
\begin{array}{ll}
\{(i, 0),(i+x, 1),(i+2 x, 2)\} & \{(i+3,0),(i+3+x, 1)\} \\
\{(i+1,0),(i+1+x, 1),(i+1+2 x, 2)\} & \{(i+4,0),(i+3+2 x, 2)\} \\
\{(i+2,0),(i+2+x, 1),(i+2+2 x, 2)\} & \{(i+4+x, 1),(i+4+2 x, 2)\} .
\end{array}
$$

Applying Theorems 3.3 and 3.5 gives the following corollary.

Corollary 3.7. Let $q$ be a prime power, $2 \leq k \leq q, 0 \leq m \leq q /(k-1)$. If there exists a $\operatorname{RTD}(k, q-m(k-2))$ then there exists a CURGDD of type $(n q(q-m(k-2)))^{k}$ with partition $2^{m n k(k-1)(q-m(k-2)) / 2} k^{n(q-m(k-2))(q-m(k-1))}$ for any integer $n$.

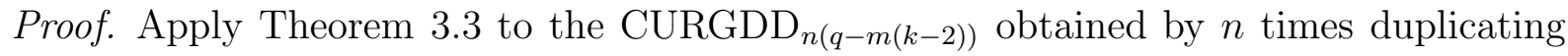
the blocks of the CURGDD $(q-m(k-2))$ constructed in Theorem 3.5.

Another corollary can be obtained from difference sets.

Corollary 3.8. If $q=2^{n}$ and there exists a CURD on $q+1$ points with partition $\prod k^{p_{k}}$ and $r$ resolution classes, then there exists a CURGDD of type

$$
\left(\frac{r\left(q^{2}-1\right)}{\operatorname{gcd}\left(r, q^{2}-1\right)}\left(\frac{q^{2}-q}{q^{2}-1}+\frac{1}{r}\right)\right)^{q^{2}+q+1}
$$


with partition

$$
2^{\left(\frac{r\left(q^{2}-1\right)}{\operatorname{gcd}\left(r, q^{2}-1\right)}\left(\frac{q^{2}-q}{q^{2}-1}+\frac{1}{r}\right)\right) q^{2} / 2} \prod k^{\left(\frac{r\left(q^{2}-1\right)}{\operatorname{gcd}\left(r, q^{2}-1\right)}\left(\frac{q^{2}-q}{q^{2}-1}+\frac{1}{r}\right)\right) p_{k}}
$$

Proof. There exists a $\left(q^{2}+q+1, q+1,1\right)$ difference set, let $B$ be the base block. We note that $B^{c}$ is a $\left(q^{2}+q+1, q^{2}, q^{2}-q\right)$ difference set. Place $\left(q^{2}-1\right) / \operatorname{gcd}\left(r, q^{2}-1\right)$ copies of the CURD on the points of $B$. Place $r / \operatorname{gcd}\left(r, q^{2}-1\right)$ copies of any 1-factorization on $B^{c}$. This gives $r\left(q^{2}-1\right) / \operatorname{gcd}\left(r, q^{2}-1\right)$ resolution classes. Develop these classes in $\mathbb{Z}_{q^{2}+q+1}$ to get a $\mathrm{CURD}_{\lambda}$ on $q^{2}+q+1$ points with partition $2^{q^{2} / 2} \prod k^{p_{k}}$ which satisfies the conditions of Theorem 3.3, where

$$
\lambda=\frac{r\left(q^{2}-1\right)}{\operatorname{gcd}\left(r, q^{2}-1\right)}\left(\frac{q^{2}-q}{q^{2}-1}+\frac{1}{r}\right) .
$$

The result follows from Theorem 3.3.

\section{Some CURGDDs}

In this section we use our constructions to produce interesting families of CURGDDs. First, restricting Theorem 3.7 to the case of CURGDDs with blocks of size 2 and 3, we derive the following corollary.

Corollary 4.1. For all $g$ and $u$ such that $g(u-1) \equiv 0 \bmod 2$ and $g u \equiv 0 \bmod 3$, except $(g, u) \in\{(2,3),(2,6),(6,3)\}, q \geq 3$ a prime power, $0 \leq m \leq q / 2$, and any integer $n$, except

$$
\begin{aligned}
(q, m, n) \in \quad & (3,1,1),(4,2,1),(11,5,1),(9,3,1),(8,2,1), \\
& (7,1,1),(6,3,2),(5,2,2),(4,1,2),(3,1,3),(4,2,3)\},
\end{aligned}
$$

there exists a CURGDD of type $(n q(q-m) g)^{u}$ with partition $2^{m n(q-m) g u} 3^{n(q-m)(q-2 m) g u / 3}$.

Proof. Apply Theorem 3.1 using a 3-RGDD of type $g^{u}$ as the master and the CURGDDs from Theorem 3.7 with $k=3$ as ingredients.

We explicitly consider a few of the many families of CURGDDs obtained from this theorem. Firstly, when $u+1=q=2^{i} \equiv 4 \bmod 6$ and $m=n=g=1$, we construct members of the extremal family meeting the first bound given in Inequality 15 and when $u-1=q=2^{i} \equiv 2 \bmod 6$ and $m=n=g=1$, we construct members of the extremal family meeting the second of these two bounds.

For $q=2^{i}, i \geq 2$, and $m=q / 4$, we obtain a family of CURGDDs with $p_{2}=3 p_{3} / 2$. This family is interesting because no CURD with blocks of size 2 and 3 can achieve this ratio due to the necessary conditions given in [4]. Next, for $q=3^{i}$, for any integer $i$, and $m=q / 3$, we have a family of CURGDDs with $p_{2}=3 p_{3}$, this family is completely known for CURDs [4]. Finally, for $q=5^{i}$, for any integer $i$, and $m=q / 5$, we have a family of CURGDDs with $p_{2}=p_{3}$. The corresponding family of CURDs was identified in [5] and remains unsolved. The next few theorems produce other members of these families that Corollary 4.1 does not. 
Theorem 4.2. There exists a CURGDD of type $(3 n)^{4}$ with partition $2^{3 n} 3^{2 n}$ and $r=6 n$ resolution classes for all $n \geq 1$.

Proof. The following is a CURGDD of type $3^{4}$ with partition $2^{3} 3^{2}$ and $r=6$. The set of points is $\mathbb{Z}_{3} \times \mathbb{Z}_{4}$, the groups are $\{(0, i),(1, i),(2, i)\}$ for $0 \leq i \leq 3$. The resolution classes are:

$$
\begin{array}{lll}
\{(i, 0),(i, 1),(i, 2)\} & \{(i+1,0),(i+2,1)\} & \{(i+1,2),(i+1,3)\} \\
\{(i+1,1),(i+2,2),(i, 3)\} & \{(i+2,0),(i+2,3)\} &
\end{array}
$$

and

$$
\begin{array}{lll}
\{(i, 0),(i+2,2),(i+1,3)\} & \{(i+2,0),(i, 2)\} & \{(i+1,1),(i+2,3)\} \\
\{(i+1,0),(i, 1),(i, 3)\} & \{(i+2,1),(i+1,2)\} &
\end{array}
$$

for $0 \leq i \leq 2$.

The following is a CURGDD of type $6^{4}$ with partition $2^{6} 3^{4}$ and $r=12$. The set of points is $\mathbb{Z}_{6} \times \mathbb{Z}_{4}$, the groups are $\{(0, i),(1, i),(2, i),(3, i),(4, i),(5, i)\}$ for $0 \leq i \leq 3$. The resolution classes are:

$$
\begin{array}{lll}
\{(i, 0),(i+1,1),(i+2,2)\} & \{(i+2,0),(i+4,1)\} & \{(i+3,1),(i+3,2)\} \\
\{(i+2,1),(i+1,2),(i, 3)\} & \{(i+3,0),(i, 2)\} & \{(i+5,2),(i+5,3)\} \\
\{(i+1,0),(i+5,1),(i+4,3)\} & \{(i+4,0),(i+2,3)\} & \\
\{(i+5,0),(i+4,2),(i+1,3)\} & \{(i, 1),(i+3,3)\} &
\end{array}
$$

and

$$
\begin{array}{lll}
\{(i+1,0),(i, 1),(i+2,3)\} & \{(i, 0),(i, 3)\} & \{(i+3,1),(i+3,3)\} \\
\{(i+2,0),(i, 2),(i+1,3)\} & \{(i+3,0),(i+3,2)\} & \{(i+2,2),(i+4,3)\} \\
\{(i+4,0),(i+1,1),(i+5,2)\} & \{(i+5,0),(i+5,1)\} & \\
\{(i+4,1),(i+1,2),(i+5,3)\} & \{(i+2,1),(i+4,2)\} &
\end{array}
$$

for $0 \leq i \leq 5$.

Applying Theorem 3.1 to these CURGDDs with RTDs as ingredients gives all the desired CURGDDs.

By applying Theorem 3.1 using the CURGDDs from Theorem 4.2 as ingredients, we obtain the following.

Corollary 4.3. If there exists a 4-RGDD of type $g^{u}$, then there exists a CURGDD of type $(3 n g)^{u}$ with partition $2^{3 n g u / 4} 3^{\text {ngu/2 }}$ for all $n \geq 1$.

Theorem 4.4. There exists a CURGDD of type $(2 n)^{5}$ with partition $2^{2 n} 3^{2 n}$ and $r=5 n$ resolution classes for all $n \geq 1$.

Proof. The following is a CURGDD of type $2^{5}$ with partition $2^{2} 3^{2}$. The set of points is $\mathbb{Z}_{2} \times \mathbb{Z}_{5}$, the groups are $\{(0, i),(1, i)\}$ for $0 \leq i \leq 4$. There are five resolution classes:

$$
\begin{array}{ll}
\{(0, i),(0,3+i),(1,4+i)\} & \{(0,1+i),(0,2+i)\} \\
\{(0,4+i),(1,1+i),(1,2+i)\} & \{(1, i),(1,3+i)\}
\end{array}
$$


for $0 \leq i \leq 4$

The following is a CURGDD of type $4^{5}$ with partition $2^{4} 3^{4}$. The set of points is $\mathbb{Z}_{4} \times \mathbb{Z}_{5}$, the groups are $\{(0, i),(1, i),(2, i),(3, i)\}$ for $0 \leq i \leq 4$. There are ten resolution classes:

$$
\{\{(j, i),(j, 4+i),(j+1,2+i)\},\{(j, 1+i),(j+2,3+i)\} \mid 0 \leq j \leq 3\}
$$

and

$$
\{\{(j, i),(j+2,4+i)\},\{(j, 1+i),(j, 3+i),(j+1,2+i)\} \mid 0 \leq j \leq 3\}
$$

for $0 \leq i \leq 4$.

Applying Theorem 3.1 to these CURGDDs with RTDs as ingredients gives all the desired CURGDDs.

Theorem 4.5. There exists a CURGDD of type $(3 n)^{5}$ with partition $2^{6 n} 3^{n}$ and $r=10 n$ resolution classes for all $n \geq 1$.

Proof. The following is a CURGDD of type $3^{5}$ with partition $2^{6} 3^{1}$ and $r=10$. The set of points is $\mathbb{Z}_{3} \times \mathbb{Z}_{5}$, the groups are $\{(0, i),(1, i),(2, i)\}$ for $0 \leq i \leq 4$. The resolution classes are:

$$
\begin{array}{lll}
\{(0, i),(1, i+1),(2, i+2)\} & \{(0, i+1),(1, i)\} & \{(0, i+2),(2, i)\} \\
& \{(1, i+2),(2, i+1)\} & \{(0, i+3),(0, i+4)\} \\
& \{(1, i+3),(1, i+4)\} & \{(2, i+3),(2, i+4)\}
\end{array}
$$

and

$$
\begin{array}{rll}
\{(0, i),(1, i+2),(2, i+4)\} & \{(0, i+2),(1, i)\} & \{(0, i+4),(2, i)\} \\
& \{(1, i+4),(2, i+2)\} & \{(0, i+1),(0, i+3)\} \\
& \{(1, i+1),(1, i+3)\} & \{(2, i+1),(2, i+3)\}
\end{array}
$$

for $0 \leq i \leq 4$.

The following is a CURGDD of type $6^{5}$ with partition $2^{12} 3^{2}$ and $r=20$. The set of points is $\mathbb{Z}_{6} \times \mathbb{Z}_{5}$, the groups are $\{(0, i),(1, i),(2, i),(3, i),(4, i),(5, i)\}$ for $0 \leq i \leq 4$. The resolution classes are:

$$
\begin{array}{lllll}
\{(0, i),(1, i+1),(2, i+2)\} & \{(1, i),(3, i+2)\} & \{(2, i+1),(4, i+2)\} & \{(2, i+3),(2, i+4)\} \\
\{(3, i),(4, i+1),(5, i+2)\} & \{(2, i),(3, i+1)\} & \{(1, i+2),(5, i+1)\} & \{(3, i+3),(3, i+4)\} \\
& \{(0, i+2),(4, i)\} & \{(0, i+3),(0, i+4)\} & \{(4, i+3),(4, i+4)\} \\
& \{(0, i+1),(5, i)\} & \{(1, i+3),(1, i+4)\} & \{(5, i+3),(5, i+4)\}
\end{array}
$$

and
$\begin{array}{lllll}\{(0, i),(4, i+2),(5, i+1)\} & \{(0, i+1),(1, i)\} & \{(1, i+1),(5, i+2)\} & \{(2, i+3),(5, i+4)\} \\ & \{(1, i+2),(2, i+1),(3, i)\} & \{(0, i+2),(2, i)\} & \{(2, i+2),(4, i+1)\} & \{(0, i+4),(3, i+3)\} \\ & & \{(3, i+1),(4, i)\} & \{(0, i+3),(3, i+4)\} & \{(1, i+4),(4, i+3)\} \\ & & \{(3, i+2),(5, i)\} & \{(1, i+3),(4, i+4)\} & \{(2, i+4),(5, i+3)\} \\ & & & & \\ \text { and } & & & & \\ & \{(0, i),(1, i+2),(2, i+4)\} & \{(1, i),(3, i+4)\} & \{(2, i+2),(4, i+4)\} & \{(2, i+1),(2, i+3)\} \\ & \{(3, i),(4, i+2),(5, i+4)\} & \{(2, i),(3, i+2)\} & \{(1, i+4),(5, i+2)\} & \{(3, i+1),(3, i+3)\} \\ & & \{(0, i+4),(4, i)\} & \{(0, i+1),(0, i+3)\} & \{(4, i+1),(4, i+3)\} \\ & & \{(0, i+2),(5, i)\} & \{(1, i+1),(1, i+3)\} & \{(5, i+1),(5, i+3)\}\end{array}$


and

$$
\begin{array}{llll}
\{(0, i),(4, i+4),(5, i+2)\} & \{(0, i+2),(1, i)\} & \{(1, i+2),(5, i+4)\} & \{(2, i+1),(5, i+3)\} \\
\{(1, i+4),(2, i+2),(3, i)\} & \{(0, i+4),(2, i)\} & \{(2, i+4),(4, i+2)\} & \{(0, i+3),(3, i+1)\} \\
& \{(3, i+2),(4, i)\} & \{(0, i+1),(3, i+3)\} & \{(1, i+3),(4, i+1)\} \\
& \{(3, i+4),(5, i)\} & \{(1, i+1),(4, i+3)\} & \{(2, i+3),(5, i+1)\}
\end{array}
$$

for $0 \leq i \leq 4$.

Applying Theorem 3.1 with RTDs as ingredients gives all the desired CURGDDs.

All CURGDDs that we have constructed thus far have had all group sizes equal. We finish by noting that CURGDDs with at least two group sizes exist.

Theorem 4.6. For all $g, u, h$ such that $g(u-1) \equiv 0 \bmod 2, g u \equiv 0 \bmod 3$, except $(g, u) \in$ $\{(2,3),(2,6),(6,3)\}$, and $0 \leq h \leq g$, there exists a CURGDD of type $(g-h)^{1} g^{u-1}$ with partition $2^{h} 3^{\text {gu/3-h}}$.

Proof. Remove $h$ points from one group of a 3-RGDD of type $g^{u}$.

Theorem 4.7. For all $g, u, h$ such that there exists a 4 - $R G D D$ of type $g^{u}$ and $0 \leq h \leq g$, there exists a CURGDD of type $(g-h)^{1} g^{u-1}$ with partition $3^{h} 3^{g u / 4-h}$.

Proof. Remove $h$ points from one group of a 4-RGDD of type $g^{u}$.

\section{Conclusion}

We have discussed only some of the families that arise from Corollary 4.1 and clearly much work has yet to be done to fully enumerate its consequences. Corollary 4.1 is derived from Theorem 3.7 by considering only $u=3$, a minor fraction of its range. Likewise, Theorem 3.7 is derived from Theorem 3.3 applied to only one class of CURGDDs coming from a particular difference construction.

The full implications of Theorem 3.3 are yet to be realized. Another similar theorem for frames appears in the sequel [3]. In fact, Theorem 3.3 can easily be modified to construct other resolvable structures. Multiple block sizes is the essential requirement, not classuniformity. For example, Uniformly resolvable designs and RRPs are ideal candidates. Further generalizations could use RGDDs as ingredients rather than RTDs.

We consider the following families of CURGDDs of particular interest and worthy of further investigation

- The extremal families for obvious reasons.

- The case $g=p_{2}$, which is not subject to the bounds from Inequality 15 . This is a subset of the more general family $g=\alpha_{l} /(k-1)$ which is not subject to the bounds from Inequalities 7 .

- The case $g=3 p_{3}$. This is in some sense complementary to the case $g=p_{2}$, but is still subject to the extremal constraints.

The sequel [3] is concerned with class-uniformly resolvable frames. There appear to be interesting geometrical constructions for CURGDDs; the authors are investigating this together with M. Greig. We believe that CURGDDS will be useful for the construction of CURDs, as already demonstrated by Lamken et al. and Wevrick and Vanstone [5, 12]. 


\section{References}

[1] A. Assaf and A. Hartman. Resolvable group divisible designs with block size 3. Discrete Math., 77:5-20, 1989.

[2] C. J. Colbourn and J. H. Dinitz, editors. The CRC Handbook of Combinatorial Designs. CRC Press, Boca Raton, 1996.

[3] P. Danziger and B. Stevens. Class-uniformly resolvable group divisible structures II: Frames. Electron. J. Combin., 11:R24, 2004.

[4] P. Danziger and B. Stevens. Class-uniformly resolvable designs. J. Combin. Des., 9:79-99, 2001.

[5] E. Lamken, R. Rees, and S. Vanstone. Class-uniformly resolvable pairwise balanced designs with block sizes two and three. Discrete Math., 92:197-209, 1991.

[6] R. Rees and D. Stinson. On resolvable group-divisible designs with block size 3. Ars Combin., 23:107-120, 1987.

[7] R. Rees and D. Stinson. On the existence of incomplete designs of block size four having one hole. Utilitas Mathematica, 35:119-152, 1989.

[8] R. Rees and D. Stinson. Frames with block size four. Canad. J. Math., 44:107-120, 1992.

[9] H. Shen. Resolvable group divisible designs with block size 4. J. Combin. Math. Combin. Comput., 1:125-130, 1987.

[10] B. Stevens and E. Mendelsohn. Packing arrays. To Appear Theoret. Comput. Sci.

[11] B. Stevens and E. Mendelsohn. Packing arrays and packing designs. Des. Codes Cryptogr., 27(1/2):165-176, 2002.

[12] D. Wevrick and S. A. Vanstone. Class-uniformly resolvable designs with block sizes 2 and 3. J. Combin. Des., 4:177-202, 1996.

[13] R. M. Wilson. Constructions and uses of pairwise balanced designs. In Combinatorics (Proc. NATO Advanced Study Inst., Breukelen, 1974) Part 1: Theory of designs, finite geometry and coding theory, pages 18-41. Math. Centre Tracts, No. 55. Math. Centrum, Amsterdam, 1974. 